\title{
PRELIMINARY MODELING FOR SOLUTE TRANSPORT IN A FRACTURED ZONE AT THE KOREA UNDERGROUND RESEARCH TUNNEL (KURT)
}

\author{
CHUNG-KYUN PARK*, JAEKWANG LEE, MIN-HOON BAIK, and JONG-TAE JEONG \\ Korea Atomic Energy Research Institute(KAERI) \\ Daedukdaero 1045, Yusung-Ku, Daejeon \\ *Corresponding author. E-mail : ckpark@kaeri.re.kr
}

Received January 26, 2011

Accepted for Publication June 08, 2011

\begin{abstract}
Migration tests were performed with conservative tracers in a fractured zone that had a single fracture of about $2.5 \mathrm{~m}$ distance at the KURT. To interpret the migration of the tracers in the fractured rock, a solute transport model was developed. A two dimensional variable aperture channel model was adopted to describe the fractured path and hydrology, and a particle tracking method was used for solute transport. The simulation tried not only to develop a migration model of solutes for open flow environments but also to produce ideas for a better understanding of solute behaviours in indefinable fracture zones by comparing them to experimental results. The results of our simulations and experiments are described as elution and breakthrough curves, and are quantified by momentum analysis. The main retardation mechanism of nonsorbing tracers, including matrixdiffusion, was investigated.
\end{abstract}

KEYWORDS : Solute Transport, Fracture, Advection, Diffusion, Organic Dye, Momentum Analysis

\section{INTRODUCTION}

A disposal research program for high level wastes was carried out at KAERI with the aim of establishing a scientific concept of geological disposal in Korea. After disposal of radioactive wastes, rock fractures will be the main flow path in deep underground areas. Groundwater will penetrate into the waste form, dissolve radionuclides, and move out through the rock fractures over the long-term. Thus, the transport of radioactive nuclides in fractured rocks is one of the important topics in safety assessment. The migration of nuclides in the rock fracture has been studied in three ways at KAERI: development of migration models, migration experiments in the laboratory under well controlled environments, and in-situ migration tests. To study real environmental conditions, KAERI constructed an underground research laboratory at its own site in Daejon, Korea, in 2007 called the KURT, which stands for the Korea Underground Research Tunnel. The main rock formation of the KURT is granite of the Mesozoic age. The access tunnel is $180 \mathrm{~m}$ with $-10 \%$ of declination. There are two research modules of about a $40 \mathrm{~m}$ length, and several research projects have been carried out in the modules.
Many countries have already run underground research laboratories to validate technologies developed in laboratories and to understand what happens under realistic conditions and environments. In particular, the Grimsel test site in Switzerland and the Äspö hard rock laboratory in Sweden have performed in-situ migration experiments with radioactive tracers [1-4]. On the other hand, in KURT it is not permitted to treat radioactive materials and only stable chemical species can be used. KAERI has carried out migration experiments with tracers in a dipole section in KURT. In order to interpret the experimental results, basic information on fracture and hydraulic properties should be known in detail. However, it is almost impossible to get information from the fracture zone, which is effectively a black box, and only local information can be acquired with sample coring and wave detectors. Therefore, because of the uncertainty in the geometry of the fracture zone in most underground laboratories, there are still many arguments on the interpretations of the experimental results. Of the early experiments carried out in fractured media, the focus of interpretation was on hydrodynamic dispersion processes and the measurement of fracture porosity or aperture $[5,6]$. The results of these experiments showed 
significant tailing in the shape of the breakthrough curves which was attributed to the arcuate shape of the flow field and the large values of dispersivity. In the later studies $[1,7]$, the experiments were interpreted with the assumption that a component of the tailing could be attributed to solute transfer from the flowing fluid into adjacent immobile zones or into the unfractured matrix. The actual mechanism of transfer has not been formally defined, although it is assumed that the exchange is an advective process whereby fluid entrapped in dead-end pores adjacent to the fracture is slightly mobile as a result of the intrusion of plumes in the fracture.

Recent approaches to model transport in fractured rocks can be classified into three groups: equivalent continuum models, fracture network models and channel network models. The continuum model characterizes hydrodynamic parameters by averaged values and has been used with transport of contaminants in porous media like soils. When the flow medium has very many interconnecting fractures, the flow medium can be treated as an equivalent porous medium $[8,9,10]$. The network models have been developed rather recently for describing rock fractures of deep underground areas more realistically $[11,12]$. In this study, a generic transport model will be developed to describe the migration of tracers in an open fracture: a variable aperture channel model for the characterization of the fracture plane, and a particle tracking scheme for solute transport. We will present the investigations of flow and migration in two-dimensions: the physical situation of the flow field and a comparison to the experiment results. The objectives of this study are to reduce uncertainty in characterizing the migration system in indefinable open fracture zones, and to generate ideas for improving the in-situ experimental system and analysis tools. As a first step, a preliminary model will be set up from the known geo-hydrological data. Second, by carrying out migration tests in the dipole region, the simulated results will be compared to the experimental data. As a study result, a revised model or assumptions will be obtained by solving discrepancies between the simulation and the tests. In addition, ideas to minimize the uncertainty in the in-situ process will be acquired.

\section{MIGRATION EXPERIMENT AT KURT}

An in-situ experimental system drilled to a fractured rock has been established in the research module at KURT. A conceptual diagram of the experimental system is depicted in Fig.1. To select a suitable water conducting fracture, several boreholes were drilled and the hydraulic characteristics of the boreholes were investigated using the BIPS (Borehole Image Processing System). The detailed information is described in other reports written in Korean [13]. The most applicable water-conducting fracture plane was selected as shown in Fig. 2; it is penetrated by the two boreholes about $2.5 \mathrm{~m}$ apart. The average aperture of the fracture measured $0.2 \mathrm{~mm}$ from the sampled cores.

One borehole was selected for injection and the other for extraction as shown in Figs.1 and 3. Tracers were put in the injection hole and pumped out at the extraction borehole with a much higher flow rate. The flow rates and other experimental conditions are arranged in Table 1. In underground environments it is difficult to get a constant flow rate even though all the experimental conditions are fixed. Moreover, sometimes unidentifiable interruptions occur causing serious effects to the test [14]. Thus, the flow rates were calculated using average values from the total eluted volume.

Three kinds of nonsorbing tracers were used: bromide as an anion, eosine and uranine as organic dyes. Uranine is the popularly known name of Na-fluorescein and tested twice as indexed TU1 and TU2 in Table 1. The eluted solutions were collected at the extraction borehole in a fraction collector, and the concentrations of the samples were analysed with an UV-VIS spectrometer for uranine and eosine, and with an electrode for $\mathrm{Br}$.

\section{CHARACTERIZATION OF THE FLOW FIELD}

As depicted in Fig. 2, the flow field seems to have a single fracture of about a $2.5 \mathrm{~m}$ distance. Since the fracture surface is rough and undulating, the aperture width varies spatially. However, there was no way to characterize the whole fracture plane; its features were only partially measurable by drilling, image processing, and so on. Therefore, there was a great degree of freedom in modeling work to describe the fracture zone, and simplification was inevitable given the complexity of these natural systems and the impossibility of complete characterization. The fracture zone is described as a two dimensional geometric field in the simulation with a variable aperture channel

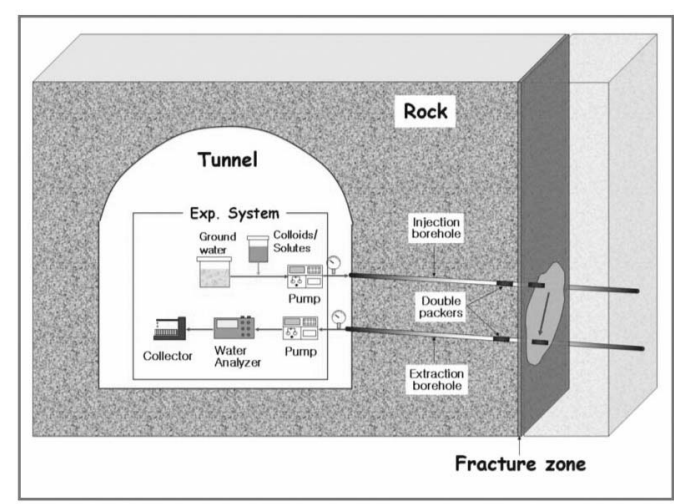

Fig. 1. Conceptual Diagram of the Experimental System at the KURT 
model $[11,15,16]$. The fracture field was divided into $\mathrm{NxN}$ (transversal x longitudinal flow direction) subsquares around the dipole.

The variation of the aperture in the fracture plane was characterized by a spatial correlation length $(\lambda)$ and an aperture density distribution. This means that for distances in the fracture plane smaller than the correlation length, the aperture values are more likely to be similar. The fracture plane was partitioned by grids with different apertures assigned to each square enclosed by grid lines. The assignment of the aperture was given by a geostatistical method that generates a two-dimensional field of a correlated distributed parameter. For the purpose of this study we chose a lognormal distribution for the variable apertures in the fracture and an exponential function for the spatial covariance of the apertures to generate different aperture widths for the square meshes composing the fracture plane. The martix decomposition method was

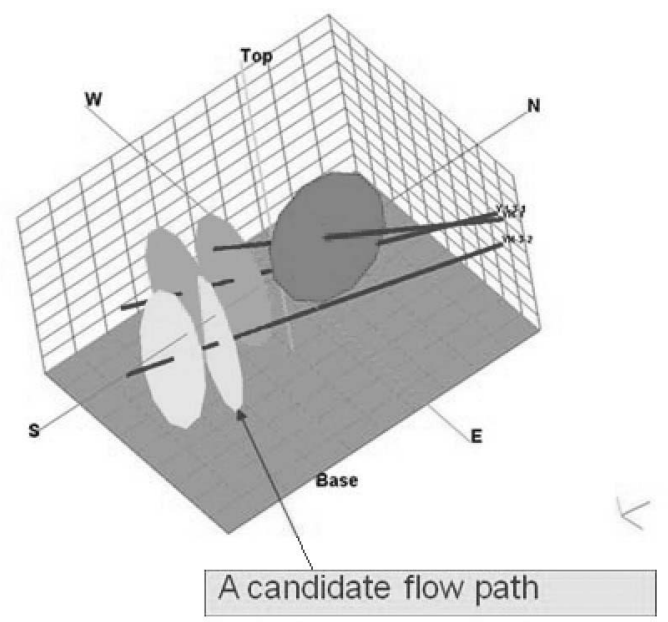

Fig. 2. Fracture Connectivity among Boreholes used to generate the lognormally distributed values of the fracture aperture. An exponential form of the covariance function was used [15].

$$
A=\sigma^{2} \exp (-2 r / \lambda)
$$

in which $\sigma^{2}$ was the variance of normal distribution of the aperture, $r$ was the separation lag, and $\lambda$ was the autocorrelation length. Fig. 4 shows the realization of the

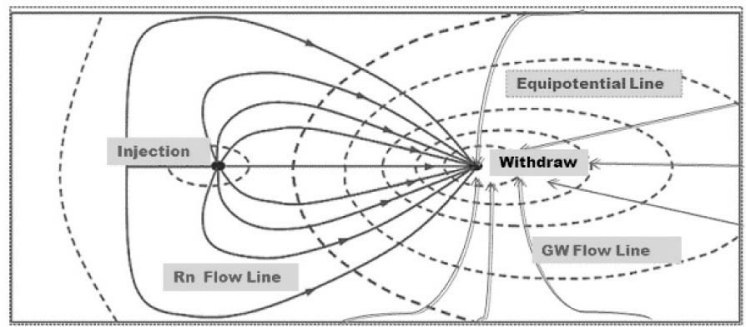

Fig. 3. Conceptual Flow Configuration of Streamlines and Equipotentials around Dipole Test Zone

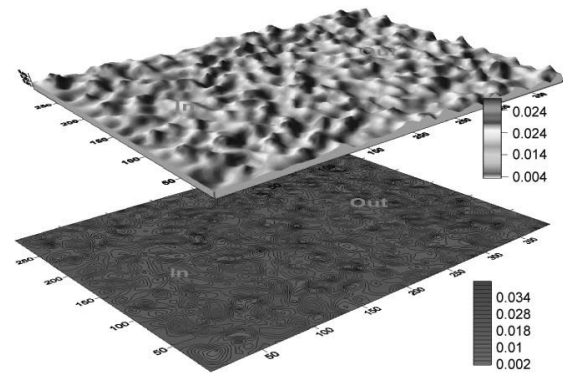

Fig. 4. Stochastically Generated Fracture Plane around the Dipole Zone ( $3 \times 4 \mathrm{~m}$ scale)

Table 1. Flow Rates and Experimental Conditions in the Migration Tests

\begin{tabular}{c|c|c|c|c|c|c}
\hline Tracer (index) & $\begin{array}{c}\mathrm{Q}_{\mathrm{in}}(\mathrm{l} / \mathrm{hr}) \\
\text { Pumping in }\end{array}$ & $\begin{array}{c}\mathrm{Q}_{\text {out }}(\mathrm{l} / \mathrm{hr}) \\
\text { Pumping out }\end{array}$ & $\begin{array}{c}\mathrm{V}_{\mathrm{tr}}(l) \\
\text { Total volume of } \\
\text { tracer }\end{array}$ & $\begin{array}{c}\text { Co(ppm) } \\
\text { Original concent. }\end{array}$ & $\begin{array}{c}\mathrm{T}(\mathrm{hr}) \\
\text { Running time }\end{array}$ & $\begin{array}{c}\mathrm{D}_{\mathrm{w}}\left(\mathrm{m}^{2} / \mathrm{s}\right) \\
\text { Diffisivity in } \\
\text { water }\end{array}$ \\
\hline Uranine1 (TU1) & 1.2 & 45.6 & 0.72 & 2,000 & 80 & $0.45 \times 10^{-9}$ \\
\hline Uranine2 (TU2) & 1.14 & 48 & 0.6 & 2,000 & 50 & $0.45 \times 10^{-9}$ \\
\hline Eosine (TES) & 1.2 & 90 & 0.72 & 1,100 & 200000 & $1.9 \times 10^{-9}$ \\
\hline Bromide (TBr) & 1.2 & 85.2 & 0.6 & - & - & - \\
\hline $\begin{array}{c}\text { Simulation } \\
\text { (SD0,SD1,SD2,SD3) }\end{array}$ & 1.2 & 50 & 0.7 & & \\
\hline
\end{tabular}


statistically generated aperture field when the mean of the lognormal aperture density distribution $v=0.02 \mathrm{~cm}$, the standard deviation of the distribution $\sigma=0.6$, and the correlation length, $\lambda=0.5$. The simulated fracture field was well developed and there was no local closed zone. The $\mathrm{Z}$-axis in the surface plot of the upper figure was exaggerated for easy understanding. And the bottom plot shows a contour map of the aperture distribution around the input and output boreholes.

\section{HYDROLOGY MODELING}

The flow system in the zone was designed as a point source-in and a point flow-out system as shown in Fig. 3, that is, a point source solution contacts at a certain point in the flow field, moves through the fracture plane, and flows out the other point of the fracture zone. It was assumed that the fracture plane was infinite and open to all directions, but for convenience, the plane had a $3 \times 4 \mathrm{~m}$ scale around the dipole in the simulation. A governing equation and boundary conditions were set for the flow system which consisted of 100x100 subsquares like finite difference meshes. The fluid flow through the fracture was then calculated for a constant flow rate as well as for constant pressure conditions.

The volumetric flow rate, $Q_{i j}$, at a subsquare $i$ can be written as $[11,16]$ :

$$
Q_{i j}=C_{i j}\left(P_{i}-P_{j}\right)
$$

where $P_{i}$ is the pressure at node $i$. Node $i$ indicates an index of the $\mathrm{i}^{\text {th }}$ subsquare in a fracture surface. $C_{i j}$ is the flow conductance between nodes $i$ and $j$.

The mass balance at each node can be written as:

$$
\sum_{j} Q_{i j}=\sum_{j} C_{i j}\left(P_{i}-P_{j}\right)=E_{i}
$$

where $E_{i}$ is the injection rate or extraction rate at node $i$. The subscript $j$ stands for the four facing nodes of the surrounding subsquares to node $i$. By rearranging the above equation for each node, a system of linear equations in the following form can be obtained:

$$
[B][P]=[E]
$$

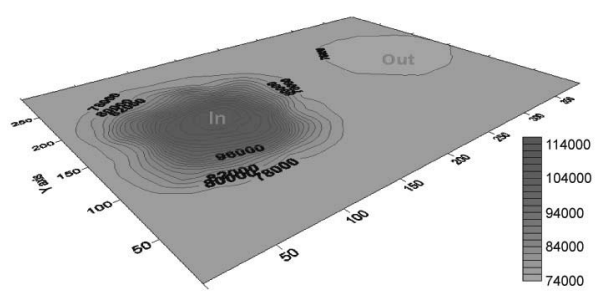

Fig. 5. Pressure Distribution in the Flow Field $(3 \mathrm{x} 4 \mathrm{~m})$ where $[\boldsymbol{B}]$ is a coefficient matrix describing the flow conductance. The matrix $[\boldsymbol{P}]$ is an array describing the pressure distribution and $[\boldsymbol{E}]$ is an array describing the net flow rates. The pressure at each node can be solved by an iteration method. Fig.5 shows the simulated pressure distribution in the flow field. The pressure built up distinctively around the injection borehole and there was negligible pressure difference around the extraction borehole, where water was pumped out more rapidly than at the inlet. The pressure $\operatorname{drop}(\Delta P)$ between the inlet and the outlet was calculated as about $6,000 \mathrm{~N} / \mathrm{m}^{2}(0.87 P S I)$. In the uranine experiment (TU2), the $\Delta P$ was about 13,700 $\mathrm{N} / \mathrm{m}^{2}$ (2 PSI). The larger $\Delta P$ in the experiment suggests that the real fracture field had a tough and undulating surface or confined fracture nets. The flow between adjacent nodes can be calculated using equation (3). The distribution of the flow vector at each point was depicted in Fig. 6. At the inlet, the flow diverged radially and converged to the outlet. At some points, there were large flow vectors where they matched the large flow conductance region. It was assumed that the fracture plane was not confined and was connected to all directions infinitely. Thus, at the four faces of the fracture plane in Figs. 5 and 6, the groundwater still came in and out. After obtaining the flow vectors at all the nodes, a solute transport could be simulated in this flow field.

\section{MODELING THE SOLUTE TRANSPORT}

A two-dimensional random-walk particle tracking algorithm was used to simulate the solute transport through the flow fields $[11,17,25]$. Four kinds of transport processes were considered: advection and longitudinal dispersion in the water conducting fracture, diffusion into the rock mass, and sorption onto the rock surface. Particle displacements at each time step consisted of an advective displacement based on the local velocities calculated using the pressure field, random diffusive displacement into the pores of the rock mass, and retardation by sorption. Particles, which represented the mass of the solute contained in a defined volume of the fluid, moved through the fracture with two types of motion. One motion was with the mean flow along the stream lines, and the other was a random

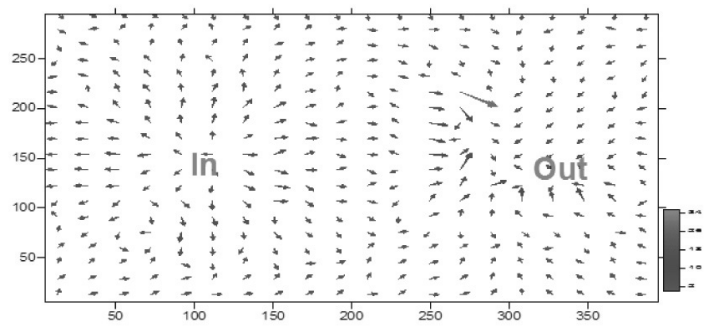

Fig. 6. Flow Vector Distribution in the Dipole Field (3x4m) 
motion, governed by a scaled probability for a matrix diffusion and sorption. At the inlet, a certain amount of the particles were introduced and distributed at each node between the flow channels with a probability proportional to the flow rates. Particles were then advected and retarded by discrete steps from node to node until they reached the outlet node at which point their arrival time was recorded. This procedure was repeated for all the particles to obtain a stable probability distribution which in turn could be regarded as an elution concentration. The residence time of a particle along each path was obtained by a sum of the residence times in all the subsquares through which a particle had passed. The migration plume could be obtained by checking the positions of the particles in the fracture surfaces at a given time.

Transport characteristics can be evaluated with a momentum analysis for elution curves $[18,19]$. The retention time $(\tau)$ was computed by the first momentum

$$
\tau=\frac{\sum t_{i} C_{i}}{\sum C_{i}}
$$

where $t_{i}$ and $C_{i}$ were a given time index $i$ and concentration at that time, respectively. Linear velocity $\left(\mathrm{v}_{\mathrm{o}}\right)$ was obtained by

$$
v_{o}=\frac{l}{\tau}
$$

Where $l$ was the distance between the inlet and the outlet. The variance of the elution curve $\left(\sigma_{t}^{2}\right)$ was given from the second central moment

$$
\sigma_{t}^{2}=\frac{\sum t_{i}^{2} C_{i}}{\sum C_{i}}-\tau^{2}
$$

From time related $\sigma_{\mathrm{t}}^{2}$ the longitudinal dispersion coefficient $\left(\sigma_{x}^{2}\right)$ was obtained by

$$
\sigma_{x}^{2}=\frac{\sigma_{t}^{2} l^{2}}{\tau^{2}}
$$

The dispersion coefficient $\left(D_{L}\right)$ was calculated by

$$
D_{L}=\frac{\sigma_{x}^{2}}{2 \tau}
$$

The dispersion length $(\alpha)$ was obtained by

$$
\alpha=\frac{D_{L}}{v_{o}}
$$

The Peclet number $\left(P_{e}\right)$ was defined as

$$
P_{e}=\frac{v_{o} l}{D_{L}}
$$

Fig. 7 shows the simulated migration plumes of a nonsorbing tracer in the fracture with time. At the early stage of migration, as shown in Fig.7(a), tracers stayed around the injection hole, but the front of the migration

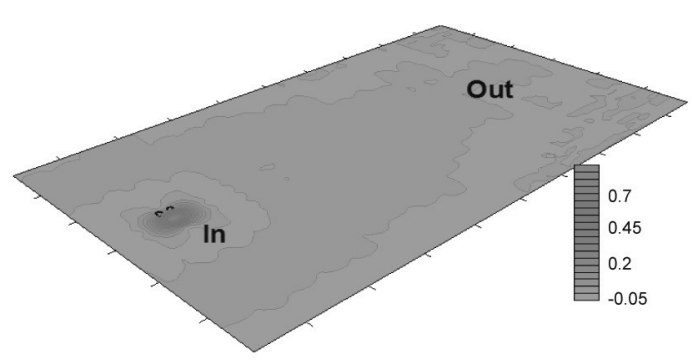

(a) Migration Plume at $20 \mathrm{~min}$

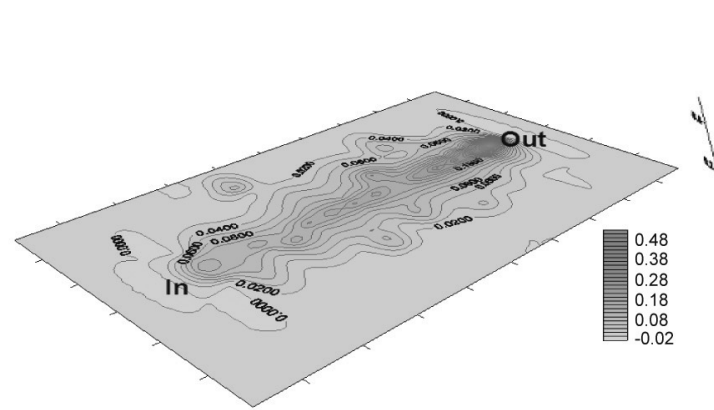

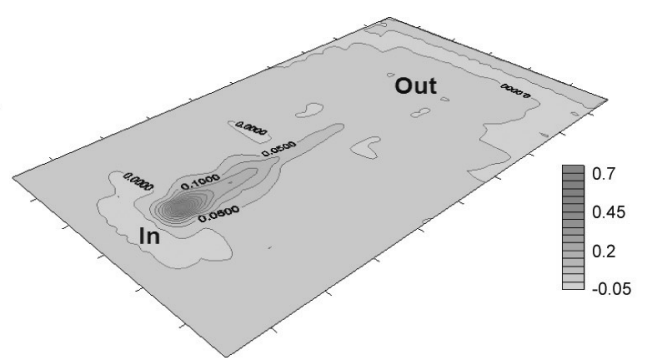

(b) $40 \mathrm{~min}$.

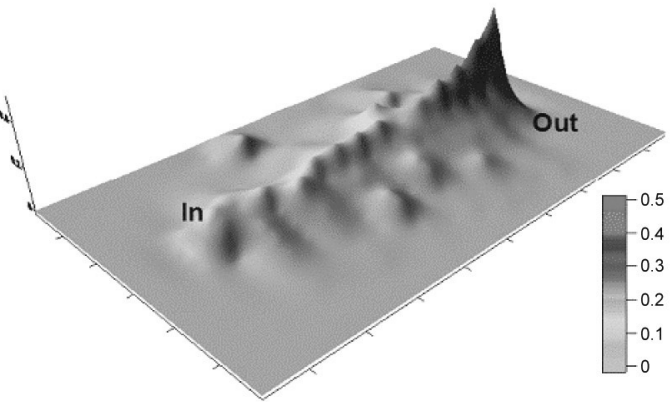

(c) Contour and Surface Plots of the Migration Plume at $80 \mathrm{~min}$

Fig. 7. Migration Plume of the Trace with Time : (a) $20 \mathrm{~min}$ (b) $40 \mathrm{~min}$ (c) $80 \mathrm{~min}$ 
plume had already spread widely all around the injection hole. A little portion of the plume moved opposite to the withdrawal hole. After 40 min., Fig. 7(b), the main plume moved directly along the straight line between the inlet and the outlet, but some portions of the plume spread in a wide region around the straight line.

Fig. 7(c) shows the contour and surface plots of the migration plume at $80 \mathrm{~min}$. After $80 \mathrm{~min}$ the main plume reached the extraction borehole, but still a large portion of the plume remained in the wide region and formed a long body. It had a long tail in the elution curve.

Fig. 8 shows the simulated elution curves of a nonsorbing tracer according to its migration characteristics at the flow field. When the tracer moved only by advection $(S D O)$ without any interactions with the fracture surface, the peak appeared after about 1.2 hours (60 $l$ of eluted volume) and the normalized concentration $\left(C / C_{o}\right)$ of the peak was 0.00175 . After 3 hours some minor peaks appeared. Those could be the long tail portion moving through tortuous long-distance paths in the dipole field as shown in Fig. 7(c). On the other hand, diffusion into rock pores can also give retardation effects and form a long tail. In order to examine the matrix diffusion effect, the elution curves were plotted together in Fig. 8 when the effective diffusivity $\left(D_{e}\right)$ varied from $3 \times 10^{-12} \mathrm{~m}^{2} / \mathrm{s}(S D 1)$ to $3 \times 10^{-10} \mathrm{~m}^{2} / \mathrm{s}(\mathrm{SD} 3)$. Those were typical values in granites $[10,20,21]$. The elution curves showed that the larger diffusivity gave the larger dispersion effects and the longer tail. The magnitude of the peak of the elution curve was also reduced. Fig. 9 shows cumulative elution curves of the nonsorbing tracer by integrating the elution curves with time. The peak heights and the recovery rates of the four cases are arranged in Table 2 . When the tracer moved only by advection $(S D O)$, the recovery rate became 0.9 , while when $D e=3 \times 10^{-12} \mathrm{~m}^{2} / \mathrm{s}(\mathrm{SD} 1)$, it became 0.82 , and when $D e=3 \times 10^{-10} \mathrm{~m}^{2} / s(S D 2), 0.56$ after 5 hours

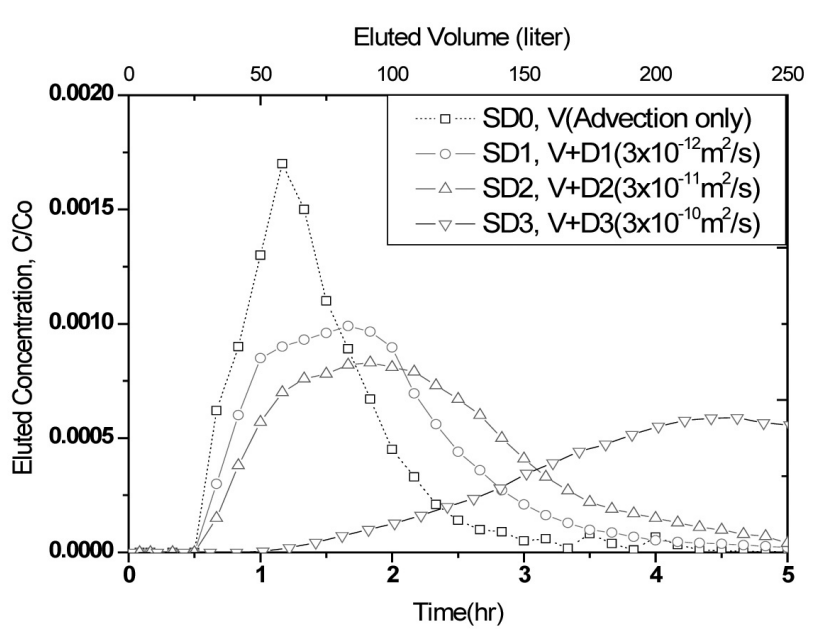

Fig. 8. Simulated Elution Curves of Nonsorbing Tracers
(250 $l$ of eluted volume). That is, a larger matrix- diffusion resulted in a larger retardation effect and a lower recovery rate. This phenomenon implies the tracer diffuses into the rock matrix and diffuses back much slower than the advection process. In order to evaluate the hydraulic and a migration properties quantitatively, a momentum analysis was carried out for the elution curves of the four cases according to equations of (5) - (11) and arranged in Table 2. The retention times $(\tau)$ were a little larger than the peak arrival times in Fig.7 due to the long tail. The values of $P_{e}$ were in the range of 5 and 13, in which spreading was caused mainly by hydrodynamic dispersion [18].

\section{EXPERIMENTAL RESULTS AND DISCUSSION}

In the underground migration process, there could be interactions of tracers with the fractured rock media. If there are interactions, the main concern would be retardation and dispersion mechanisms in the process. To deal with such an occurrence,, we examined the experimental data for three topics: hydrodynamic properties by momentum analysis, matrix-diffusion effects by comparison with the simulated ones, and multi-channel path effects by analyzing elution curves.

\subsection{Flow Stability and Multi-channel Path}

The experimental curves of the conservative tracers were plotted together as a function of the eluted volume to compensate for the difference of the flow rates in Fig. 10. A small front peak appeared before the main peak in the elution curves of uranine1 (TU1) and eosin (TES), but it did not seem to be the fastest path in the flow field because the other curves of uranine2 (TU2) and bromide (TBr)

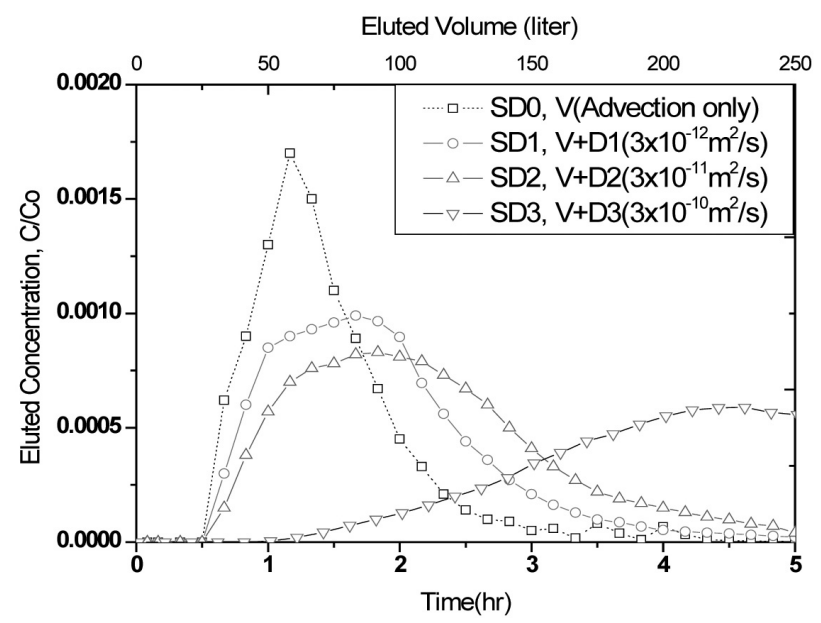

Fig. 9. Simulated Cumulative Elution Curves of Nonsorbing Tracers 
did not show such a trend. It may have originated from a flow disruption and an unstable state of the system. It is not easy to trace the cause of the flow disruption, but gas bubbles are considered one of the potential sources. Generally, some portion of dissolved gas in groundwater can form gas bubbles by a change of environmental conditions in rock fractures or flow lines. The bubbles could plug a flow path or tube lines and give a pressure build-up in the process. The flow of the tracer might be interrupted for a while at the first peak time and then flow again. When it flows again, the tracer moves faster to release the pressure build-up. Thus, there are not enough opportunities to diffuse into the rock mass and interact with the fracture surface. The greater peak height of $T U 1$ than the others seems to indicate such an effect.

The other interesting phenomenon is that the strong secondary peak appeared a long time after the first main plume in Fig. 10. Fig.11 shows the cumulative elution curves by summing up the elution curves with time. In the Figs. 10 and 11 there is no remarkable increase of concentration for a long while between roughly $300 l$ and $2000 l$ of the eluted volume. Such a trend means that there is a tortuous path from the inlet to the outlet and the plume through this path comes out at a later stage. The internal

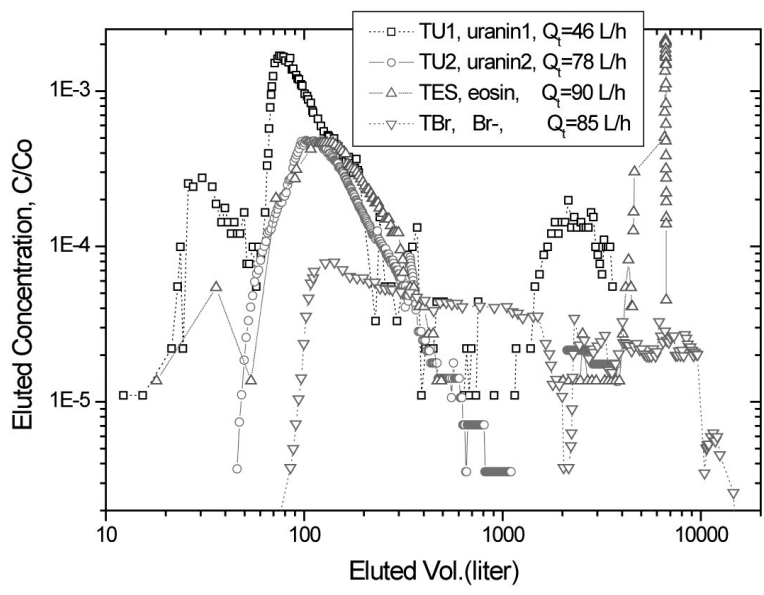

Fig. 10. Experimental Elution Curves of the Nonsorbing Tracers

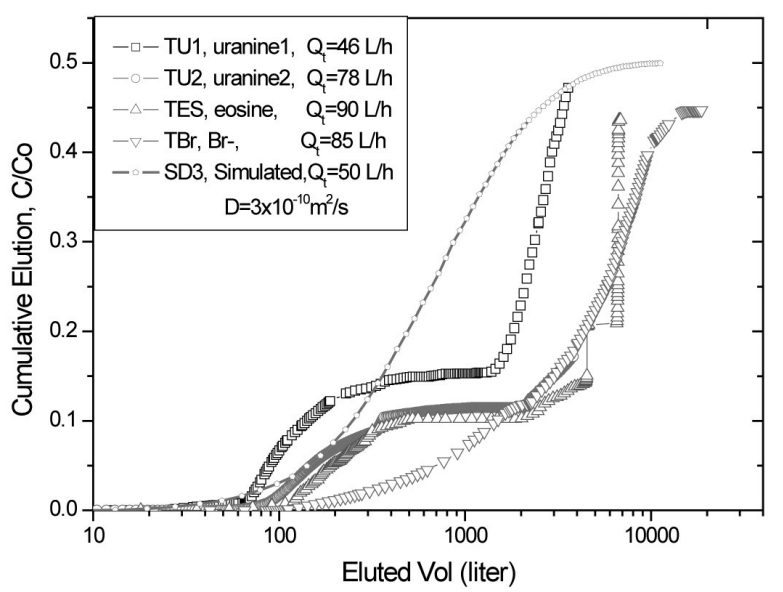

Fig. 11. Cumulative Elution Curves

Table 2. Momentum Analysis for the Elution Curves

\begin{tabular}{c|c|c|c|c|c|c|c|c}
\hline \multirow{2}{*}{ parameter } & \multicolumn{4}{|c}{ Simulation } & \multicolumn{4}{c}{ Experiment } \\
\cline { 2 - 10 } & SD0 & SD1 & SD2 & SD3 & TU1 & TU2 & TE & Br \\
\hline$\tau(h r)$ & 1.43 & 1.77 & 2.16 & 2.7 & 2.31 & 4.77 & 4.38 & 0.57 \\
\hline $\mathrm{v}_{\mathrm{o}}(m / h)$ & 1.75 & 1.42 & 1.15 & 0.8 & 1.08 & 0.52 & 0.08 \\
\hline$\sigma_{\mathrm{t}}^{2}$ & 0.33 & 0.52 & 0.91 & 1.58 & 1.44 & 80.1 & 93.6 & 1391 \\
\hline$\sigma_{\mathrm{x}}^{2}$ & 0.99 & 1.04 & 1.21 & 2.14 & 1.69 & 22.0 & 30.4 & 8.4 \\
\hline $\mathrm{D}_{l}$ & 0.35 & 0.3 & 0.28 & 0.39 & 0.37 & 2.31 & 3.47 & 0.13 \\
\hline$\alpha$ & 0.2 & 0.21 & 0.24 & 0.49 & 0.34 & 4.41 & 6.09 & 1.67 \\
\hline $\mathrm{P}_{\mathrm{e}}$ & 12.5 & 11.9 & 10.3 & 5.0 & 7.34 & 0.57 & 0.41 & 1.49 \\
\hline $\mathrm{F}^{1)}$ & 0.9 & 0.82 & 0.75 & 0.56 & $0.5+$ & $0.18+$ & $0.45+$ & 0.45 \\
\hline $\mathrm{H}\left(\mathrm{C} / \mathrm{C}_{\mathrm{o}}\right)^{2)}$ & $1.7 \times 10^{-3}$ & $1 \times 10^{-3}$ & $8 \times 10^{-4}$ & $6 \times 10^{-4}$ & $1.7 \times 10^{-3}$ & $5 \times 10^{-4}$ & $5 \times 10^{-4}$ & $8 \times 10^{-5}$ \\
\hline
\end{tabular}

${ }^{1)} \mathrm{F}$ : recovery after 5 hours ( $250 \mathrm{l}$ of eluted volume) in the simulation and after more than 50 hours in the tests.

${ }^{2)} \mathrm{H}\left(\mathrm{C} / \mathrm{C}_{\mathrm{o}}\right)$ : normalized peak height 
structure of the flow fracture should be more complex than the model assumption : an infinite single fracture having variable apertures. Even though each path has a certain amount of diffusion, the channeling effects dominated the overall elution in this system.

\subsection{Dispersion Mechanism and Matrix-diffusion}

The experimental curves are plotted all together with the simulated ones in Fig. 12. The characteristics of the peak of TU1 showed a trend similar to the simulated advection-only case $(S D O)$. As mentioned in section 6.1, it is reasonable to conclude that the main plume of TU1 transported by advection without noticeable matrixdiffusion. The uranin2 (TU2) and the eosine (TES) curves were a similar to each other: a remarkable retardation effect and smaller peak height than TU1. The recovery rates of the TU2 and TES were also lower than that of TU1 with time as shown in Fig. 11. Since the SD3 shows an elution trend similar to TU2 and TES in Fig. 12, it seems that they moved with $D_{e}=3 \times 10^{-10} \mathrm{~m}^{2} / \mathrm{s}$, which has a somewhat higher value in crystalline rocks. Vilks et al. [20] conducted an in- situ diffusion test in granite and got $D_{e}$ for $\mathrm{I}^{-}$from $2 \times 10^{-14} \mathrm{~m}^{2} / \mathrm{s}$ to $1.9 \times 10^{-13} \mathrm{~m}^{2} / \mathrm{s}$. In the laboratory tests the values of $D_{e}$ were in the order of $10^{-12} \mathrm{~m}^{2} / \mathrm{s}$ [20, 21, 26]. In those in-situ and laboratory tests the tracers were in contact with fresh rock bodies. Novakowski et al.[23] performed a migration test with nonsorbing tracers in a dolostone fracture with the mean aperture, $b=0.5 \mathrm{~mm}$ and the flow rate, $Q=150 \mathrm{l} / \mathrm{hr}$. They obtained a high diffusivity of $D_{e}=3 \times 10^{-}$ ${ }^{10} \mathrm{~m}^{2} / \mathrm{s}$ and the recovery percent was in the range of $36 \%$ and $62 \%$. They concluded that the matrix diffusion plays a great role in the transport process. Andersson et al.[10] also obtained high $D_{e}$ for $\mathrm{Br}^{-}$as $D_{e}=9 \times 10^{-10} \mathrm{~m}^{2} / \mathrm{s}$ in Äspö diorite. They explained that the high $D_{e}$ value was not

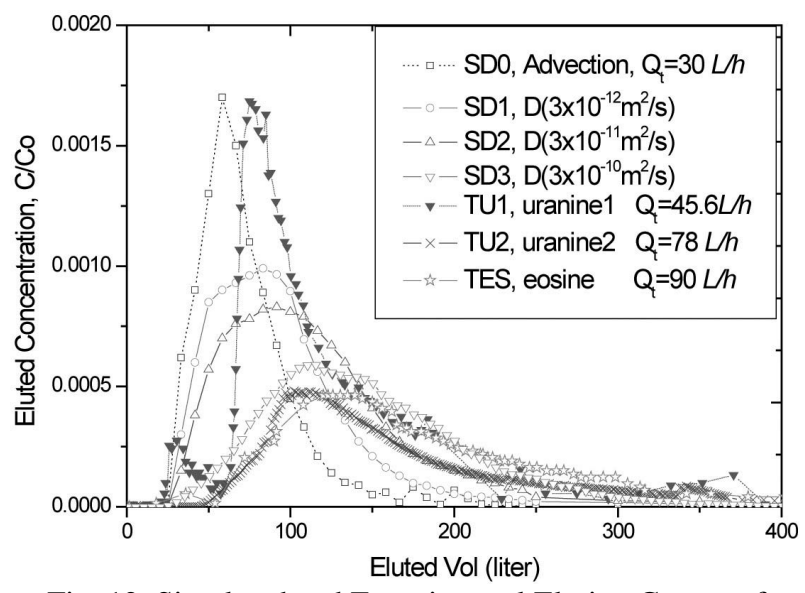

Fig. 12. Simulated and Experimental Elution Curves of Nonsorbing Tracers attributed to the rock matrix, but to the diffusion into the fault gauge materials. Therefore, the tracers diffuse not only into the rock mass from the fracture surface, but also into the fracture filling materials in the migration process, and such behavior gives great retardation effects.

The peak of the anion, $\mathrm{Br}(\mathrm{TBr})$, came later and smaller than the others. This delay could be attributed to the difference in diffusivity. The larger diffusivity of $\mathrm{Br}^{-}$in Table 1 resulted in a deeper penetration into the rock mass and gave a delayed return to the fracture. The same phenomenon was observed by Novakowsky et al.[23]: the $\mathrm{Br}^{-}$curve shifted backwards compared to lissamine, an organic dye.

\subsection{Elution Characteristics and Recovery}

The $P_{e}$ numbers of all the simulated curves in Table 2 indicate that hydrodynamic advection was the dominant flow system rather than diffusion [18]. The difference of $P_{e}$ between the simulated SD3 and the experimental TU2 and TES having a similar elution trend was caused by the large later peak in the tests. If the dipole region has a single open channel as in the model, the retention time $(\tau)$ will be about 1.43 hour for only the advection case, or 2.7 hour for the highly diffused case. On the other hand, the retention times in the experiments had much larger values due to the tortuous multi-channel flow. Most of the experiments ran long enough to recover the tracers as shown in Table 1 and Fig. 11. A significant increase of recovery, even for much extended running time, was not expeted. Thus, we regarded the unrecovered portion as having already flowed out to the outer environment. If there is a large scale flow potential or a background flow around the dipole region, some portion of the tracer could be captured by the background flow and might not reach the withdrawal hole. In the simulation, background flow was not assumed.

The preliminary simulation and the tests suggested that the following points should be considered for the next step test: First, increase the recovery rate by adjusting the experiment systems, for example, changing the inlet and outlet boreholes, increasing the extraction rate, and testing in new boreholes. Second, check for external interruptions by continuously measuring pressures at the inlet and outlet.

\section{CONCLUSIONS}

The developed variable aperture channel model was used successfully to characterize a flow field in a fracture zone, and also the particle tracking scheme well described the migration plumes and elution curves of tracers through a fracture zone. The simulated plumes did not move through a straight line between the inlet and the outlet in the fracture, rather they spread widely along tortuous paths by following the path of least resistance. In the experiment, a strong later peak appeared through a tortuous path after all of the first 
main peak disappeared. When the recovery of the tracer is low, the experiment should be run longer to check tortuous flow paths or multi-channel effects. The matrixdiffusion into the rock mass greatly affected the retardation of the nonsorbing tracers in this granite fracture. The results of the preliminary modeling suggest the following for future tests: First, increase the recovery rate by adjusting the experimental systems, such as changing the inlet and outlet boreholes, increasing the extraction rate, or testing in new boreholes. Second, check for external interruptions by continuously measuring the pressure at the inlet and outlet. In revising the model, the assumption of the open single fracture should reflect the multi-channel flow.

\section{NOMENCLATURE}

$Q_{i j} \quad$ volumetric flow rate from $\mathrm{i}$ to $\mathrm{j}(\mathrm{l} / \mathrm{hr})$

$P_{i} \quad$ pressure at node $i$,

$C_{i j} \quad$ flow conductance between nodes $i$ and $j$.

$E_{i}$ injection rate or extraction rate at node $i$.

$[B]$ coefficient matrix describing the flow conductance.

$[P]$ array describing the pressure distribution and

$[E]$ array describing the net flow rates.

$\sigma^{2} \quad$ variance of normal distribution of the aperture,

$r \quad$ separation lag

$\lambda$ autocorrelation length

$\tau$ retention time $(h r)$

$C_{i}$ concentration at a certain time $t_{i}$,

$\mathrm{v}_{\mathrm{o}} \quad$ Linear velocity $(\mathrm{m} / \mathrm{hr})$

$l \quad$ distance between the inlet and the outlet $(m)$

$\sigma_{\mathrm{t}}^{2} \quad$ variance of the elution curve

$\sigma_{\mathrm{x}}^{2} \quad$ longitudinal dispersion coefficient

$D_{e} \quad$ effective diffusivity $\left(\mathrm{m}^{2} / \mathrm{s}\right)$

$D_{L} \quad$ dispersion coefficient $\left(\mathrm{m}^{2} / \mathrm{s}\right)$

$\alpha \quad$ dispersion length $(m)$

$P_{e} \quad$ Peclet number

\section{SUBSCRIPT}

$i \quad$ index of the $\mathrm{i}^{\text {th }}$ subsquare in a fracture surface

$j \quad$ four facing nodes of the surrounding subsquares to node $i$

\section{REFERENCES}

[1] J. Hadermann, "The Grimsel Migration experiment : integrating field experiments, laboratory investigations and modelling", J. of Cont. Hydrol. 21, p.87-100 (1996)

[ 2 ] B.Kienzler, Vejmelka, P., Römer, J., Fanghänel, E, "SwedishGerman actinide migration experiment at Äspö HRL.", Journal of Contaminant Hydrology 61, p.219- 233 (2003)

[3 ] P.Smith, W.Alexander, W.Kickmaier,K.Ota, B.Frieg, I.McKinley, "Development and testing of radionuclide transport models for fractured rock", J. of Cont. Hydrol. 47, p.335 (2001)

[4] A.Mori, W.Alexander, H.Geckeis, W.Hauser,T.Schafer, J.Eikenberg, Th.Fierz, C.Degueldre, T.Missana, "The colloid and radionuclide retardation experiment at the Grimsel Test Site”, Colloids and Surfaces A: Physicochem.
Aspects, 217, p.33 (2003)

[5] Y.W. Tsang, "Usage of equivalent apertures for rock fractures as derived from hydraulic and tracer tests", Water Resour. Res., 28(5), p.1451 (1992)

[6] L. Moreno, I. Neretnieks, and C.Klockars, "evaluation of some tracer tests in granitic rock at Finnsjon", SKB TR83-38, (1983).

[7] M.Becker and A. Sapiro, "tracer transport in fractured crystalline rock ; evidence of nondiffusive breakthrough tailing", Water Resour.Res.36, p.1677 (2000)

[ 8 ] A. Jacob, M.Mazurek, and W.Heer, "solute transport in crystalline rocks at Aspo-II", J. of Cont. Hydrol.61, p. 175(2003)

[9] R.McLaren, P.Forsyth, E.Sudicky, J.VanderKwaak, F.Schwartz, and J.Kessler, "Flow and transport in fractured tuff at Yucca Mountain”, J. of Cont. Hydrol. 43, p. 211(2000)

[10] P. Andersson, J.Byegard, E.Tullborg, T.Doe, J.Hermanson, and A.Winberg, "In situ tests to determine retention properties of a block scale fracture network at the Aspo HLR, Sweden”, J. of Cont. Hydrol. 70, p. 271(2004)

[11] L. Moreno and I. Neretnieks, "Flow and nuclide transport in fractured media," J. of Contaminant Hydrology, 13, p.49-71 (1993).

[12] A.Steele and D.Lerner, "predictive modelling of NAPL injection tests in variable aperture spatially correlated fractures", J. of Cont. Hydrol. 49, p. 287 (2001)

[13] M.H.Baik, C.K.Park, J.K.Lee, A study on the nuclide migration and retardation in natural barriers, KAERI/RR$3114 / 2009$ (in Korean)

[14] B. Kienzler, Vejmelka, P., Römer, J., Schild,D.,and Jansson, M.. " Actinide migration in fractures of granite host rock: laboratory and in situ investigations", Nuclear Technology 165 , p.223 (2009)

[15] A.J. Desbarats, "Macrodispersion in Sand-Shale Sequences", Water Resour. Res., 26 (1), p.153, (1990).

[16] C.K.Park, D.K.Keum and P.S.Hahn, "Stochastic analysis of contaminant transport through a rough-surfaced fracture", Korean J. of Chem. Eng., 12, 428 (1995)

[17] C.K.Park, T. T. Vandergraaf, D. J. Drew, and Hahn, P.S., "Analysis of the Migration of nonsorbing tracers in a natural fractures in granite using a variable aperture channel model", J. of Cont. Hydrol. 26, p. 97 (1997)

[18] J. Seinfeld, and L. Lapidus, Process modeling, estimation, and identification, p.161-164, Prentice Hall (1974)

[19] P. Vejmelka, Kienzler, B. Roemer, J.; Ch. Marquardt, E. Soballa, F. Geyer, T. Kisely, D.Heathman, Actinide migration experiment in the HRL Aspo, Sweden: results of laboratory and in situ experiments (Part I). Wissenschaftliche Berichte, FZKA-6652 92001)

[20] P.Vilks, J.Cramer, M.Jemsen, N.Miller, H.Miller, F.Stanchell, "In situ diffusion experiment in granite", J. of Contaminant Hydrology, 61, p.191, (2003).

[21] C.K.Park, M.H.Baik, "Diffusion of some chemical species through a granite,” Kor. J. of Chem. Eng., 26(5), p.1279 (2009)

[22] J.Bear, Hydraulics of Groundwater, p.237, McGraw-Hill Inc. (1979)

[23] K.Novakowski, G.Bickerton, P.Lapcevic, "Interpretation of injection-withdrawal tracer experiments conducted 
between two wells in a large single fracture", J. of Cont. Hydrol. 26, p. 97 (2004)

[24] R. Haggerty, S.Fleming, L.Meigs, S.McKenna, “Tracer test in fractured dolomite: 2. Analysis of mass transfer in single well injection-withdrawal tests", Water Resour. Res. 37, p.1129 (2001)
[25] L.Pan and G.Bodvarsson, "Modeling transport in fractured porous media with the random-walk particle method", Water Resour. Res. 38, p.1029 (2002)

[26] H. Sato, "matrix diffusion of simple cations, anions, and neutral species in fractured crystalline rocks", Nuclear Technology, 127, p.199 (1999) 